\title{
Unified Simulation of Transport and Luminescence in Optoelectronic Nanostructures
}

\section{Journal Article}

Author(s):

Steiger, Sebastian; Veprek, Ratko G.; Witzigmann, Bernd

Publication date:

2008

Permanent link:

https://doi.org/10.3929/ethz-b-000014891

Rights / license:

In Copyright - Non-Commercial Use Permitted

Originally published in:

Journal of Computational Electronics 7(4), https://doi.org/10.1007/s10825-008-0261-z 


\title{
Unified simulation of transport and luminescence in optoelectronic nanostructures
}

\author{
Sebastian Steiger • Ratko G. Veprek • \\ Bernd Witzigmann
}

Published online: 1 October 2008

(C) Springer Science+Business Media LLC 2008

\begin{abstract}
Computer simulation of microscopic transport and light emission in semiconductor nanostructures is often restricted to an isolated system of a single quantum well, wire or dot. In this work we report on the development of a simulator for devices with various kinds of nanostructures which exhibit quantization in different dimensionalities. Our approach is based upon the partition of the carrier densities within each quantization region into bound and unbound populations. A bound carrier is treated fully coherent in the directions of confinement, whereas it is assumed to be totally incoherent with a motion driven by classical drift and diffusion in the remaining directions. Coupling of the populations takes place through electrostatics and carrier capture. We illustrate the applicability of our approach with a wellwire structure.
\end{abstract}

Keywords Nanostructures - Optoelectronics - Transport · Luminescence · Quantum wells · Quantum wires

\section{Introduction}

Nanostructures have become an indispensable part of today's consumer technology in the form of quantum-well (QW) lasers, light-emitting diodes (LEDs), or high electron mobility transistors (HEMTs). Moreover, a plethora of possible future devices like nanowire transistors [1] and solar cells [2], quantum wire (QWR) [3], dot (QD) [4] or photonic crystal [5] low-threshold lasers as well as applications

S. Steiger $(\varangle) \cdot$ R.G. Veprek · B. Witzigmann Integrated Systems Laboratory, Department of Information

Technology and Electrical Engineering, ETH Zurich,

Gloriastrasse 35, 8092 Zurich, Switzerland

e-mail: steiger@iis.ee.ethz.ch in quantum information science [6] assert a constant stimulus within the academic community. Computer simulation has proven in the past to be able to contribute to the development process by gaining understanding about the fundamental processes governing the behaviour of a structure. Designs are optimized in an easy, fast and inexpensive way, thus avoiding sometimes cumbersome and expensive fabrication in the lab.

Research in carrier transport simulation is currently centered either on experimental nanodevices with near-ballistic [7] or one-dimensional [8] transport using quantum models, or on industrial devices using classical or quasi-classical models [9]. In optoelectronics, focus is put on predicting accurate gain [10] and luminescence [11] spectra from quantum theory, but transport through the device is mostly carried out in a classical picture [12]. The underlying reason for this conceptual inconsistency is the intricate situation in a quantum well, wire or dot light emitter: gain spectra have clear indications of low-dimensional quantum states, but carrier injection is highly diffusive due to high temperatures and carrier densities as well as the long travelling distance of a particle before it recombines (as opposed to, for example, photoluminescence experiments). These opposites suggest that a semi-coherent microscopic model would be best suited. However, the only two quantum formalisms capable of such a treatment (the nonequilibrium Green function (NEGF) [13] formalism and the Wigner function formalism [14]) are out of reach from a numerical point of view when the device is nonplanar and/or a few microns big.

In this contribution we report on the development of a new simulator aimed at applications in optoelectronics. It is based on a mixed picture where carriers are described quantum-mechanically in some directions and classically in others. The fundamental concept we follow is that within 
a nanostructure, the carrier population partitions into carriers which have sufficient energy to leave the structure and those which are confined to the structure. The latter population can only move in the unconfined directions (two in a quantum well, one in a quantum wire, zero in a quantum dot) and experiences enough scattering such that transport in these directions is governed by drift and diffusion. On the other hand, much less scattering occurs in the confined directions because of their small extensions and modified density of states, and the carrier is best described by its quantum-mechanical wave function and energy which we determine by means of the popular $\mathbf{k} \cdot \mathbf{p}$ method [15].

Rigorous adherence to this approach has the advantage that it can be applied to structures which contain a combination of multiple wells, wires and dots. This is an uncommon feature. The different transport problem dimensionalities of the various populations lead to a system of coupled transport equations defined on a diversity of spatial grids.

A similar approach to transport in nanostructures has been presented in [16] in the sense that there is a $\mathbf{k} \cdot \mathbf{p}$ Schrödinger problem solved together with a drift-diffusion equation. However, our method differs in the assumption that wavefunctions describe only bound carriers in the directions where they are localized. They enter the transport problem indirectly via their influence on the electrostatic potential and recombination rates. The quasi-Fermilevel, on which the current in both our model and the model in [16] depends, is in our case not calculated from the quantum results. We believe that a purely classical picture is more suitable for carriers in large bulk regions. Our model thus assumes that behaviour in such regions is governed by local rather than nonlocal effects, hence restricting the computationally expensive nonlocal eigenvalue (Schrödinger) problems to the central parts of the device.

Limitations of our model lie in the assumption of type I heterojunctions, equilibrium distributions within a carrier population and in the strict division between coherent and incoherent directions. Furthermore, we consider only steady-state transport and isothermal situations with a globally constant temperature, although the latter limitation could be overcome at a later stage.

The aim of this paper is twofold: In Sects. 2-4 the reader is introduced to the model with the purpose of giving the complete set of transport (Sect. 2) and luminescence (Sect. 3) equations entering our calculation as well as some essential numerical considerations (Sect. 4). As the power of a physical model in the end is always determined by its ability to describe real-world measurements, a first simulation example of an experimentally realized well-wire structure [17] is given in Sect. 5 .

\begin{tabular}{lcccc} 
Table 1 & Problem dimensionalities for different nanostructures \\
\hline & Bulk & Well & Wire & Dot \\
\hline Transport & 3D & 2D & 1D & - \\
Wavefunction & - & 1D & 2D & 3D \\
\hline
\end{tabular}
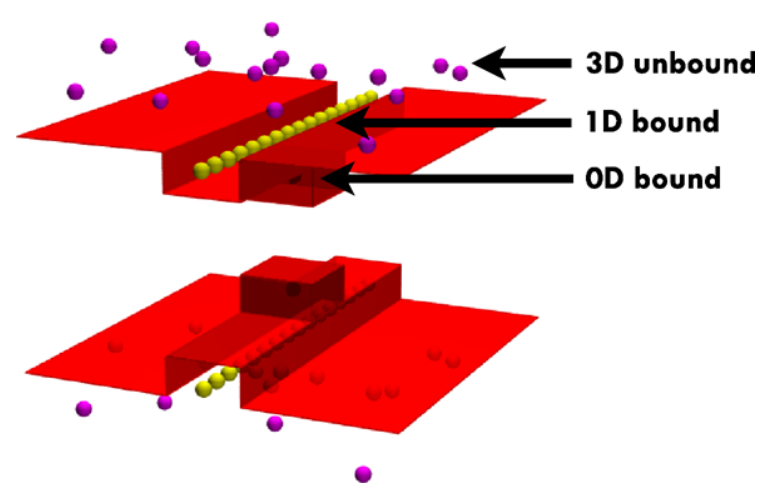

Fig. 1 Sketch of bound and unbound carrier populations

\section{Transport in low-dimensional structures}

Consistent partitioning into energetically bound and unbound carrier populations leads to the problem dimensionalities given in Table 1. Each population has a separate continuity equation and a separate Schödinger equation in our model. The populations are coupled through the electrostatic potential, which is always solved on the bulk grid, and through capture of carriers from high-dimensional into lowdimensional populations. The charge density entering the Poisson equation (10) includes the low-dimensional densities which are spread out according to their wavefunction in the quantized directions. Capture acts as an outflow (recombination) on the high-dimensional grid and as an inflow (generation) on the low-dimensional grid. Figure 1 gives a schematic view of the situation.

\subsection{Continuity equation}

In the drift-diffusion model, carrier transport is determined by the zeroth moment of the Boltzmann equation:

$$
\nabla \cdot \mathbf{J}_{n}-\frac{\partial n}{\partial t}=R_{n}-G_{n}, \quad \nabla \cdot \mathbf{J}_{p}-\frac{\partial p}{\partial t}=R_{p}-G_{p} .
$$

$n$ and $p$ are the electron or hole particle densities, $R_{n, p}$ and $G_{n, p}$ denote recombination and generation, and $\mathbf{J}_{n, p}$ is the particle current given by

$\mathbf{J}_{n}=\mu_{n} n \nabla E_{F n}, \quad \mathbf{J}_{p}=\mu_{p} p \nabla E_{F p}$,

where $\mu_{n, p}$ are the mobilities and $E_{F n, p}$ the quasi-Fermilevels (QFL). Validity of a generalized Einstein relation [18] between drift and diffusion processes at nonequilibrium is 
tacitly assumed. Consistent with our classical picture, a single parabolic band is assumed as dispersion relationship leading to

$n_{d}=N_{c}^{(d)} \mathcal{F}_{d / 2-1}\left(\frac{E_{F n}-E_{c}+e \phi}{k T}\right)$,

$p_{d}=N_{v}^{(d)} \mathcal{F}_{d / 2-1}\left(\frac{E_{v}-e \phi-E_{F p}}{k T}\right)$.

Here $\mathcal{F}_{v}$ stands for the Fermi integral of order $v, E_{c, v}$ denote the conduction and valence band edges, $e$ is the positive elementary charge, $\phi$ is the electrostatic potential and $T$ is the temperature. The index $d$ marks the dimensionality of the carriers which affects the order of the Fermi integral as well as the effective densities of states $N_{c, v}$ :

$N_{c, v}^{(d)}=S^{(d)} \Gamma(d / 2)\left(\frac{\sqrt{2 m_{e, h} k T}}{2 \pi \hbar}\right)^{d}$,

where $m_{e, h}$ are effective masses fitted to the density of states at the band edge, $S^{(d)}$ is the volume of the $d$-dimensional sphere and $\Gamma$ the Euler Gamma function. Taking the Boltzmann limit of (3) and substituting everything into (2) leads to

$$
\begin{aligned}
& \mathbf{J}_{n}=\mu_{n}\left(n \nabla\left(E_{c}-e \phi-\frac{d}{2} k T \log m_{e}\right)+k T \nabla n\right), \\
& \mathbf{J}_{p}=\mu_{p}\left(p \nabla\left(E_{v}-e \phi+\frac{d}{2} k T \log m_{h}\right)-k T \nabla p\right) .
\end{aligned}
$$

A materialwise constant mobility and the temperatureand doping dependent Arora mobility model [19] are currently considered.

In addition to the drift and diffusion currents, a change in bandgap or mass leads to corresponding currents. We model these quantities in a continuous way. The given model naturally takes into account heterojunctions based on the validity of (2) even for spatially varying band edges and masses. Other widely used heterojunction models $[20,21]$ are restricted to situations where the current essentially flows perpendicular to the junction and cannot be applied to intersections of three or more materials.

\subsubsection{Recombination mechanisms}

Our model employs the standard Shockley-Read-Hall (SRH), Auger and radiative recombination mechanisms [19]. In the latter, an alternative to the standard approach was implemented in which recombination is calculated from the luminescence as described in Sect. 3.2, without the need of an additional material parameter. Doping and temperature dependence was accounted for in the SRH minority carrier lifetimes according to

$\tau_{S R H}=\frac{\tau_{\max }}{1+\left(\frac{N}{N_{r e f}^{S R H}}\right)^{\gamma}} \cdot\left(\frac{T}{300 \mathrm{~K}}\right)^{T_{\alpha}}$.

\subsubsection{Carrier capture}

Capture acts as a recombination mechanism on the higherdimensional grid of the unbound population and as a generation mechanism on the lower-dimensional grid of the bound population. As shown in Sect. 4.2, a geometrical factor enters due to the different dimensionalities of the carriers. Depending on the structure, capture can happen between any combination of bulk, well, wire and dot populations. The model employed has been developed for quantum wells [22] using equilibrium scattering rates, but the derivation can be straightforwardly extended to wires and dots. The net capture rate is given by

$R_{c n}=\frac{n_{d}}{\tau}\left(1-\frac{n_{d-1}}{N_{d-1}}\right)\left(1-\exp \left(\frac{E_{F n, d-1}-E_{F n, d}}{k T}\right)\right)$,

$R_{c p}=\frac{p_{d}}{\tau}\left(1-\frac{p_{d-1}}{P_{d-1}}\right)\left(1-\exp \left(\frac{E_{F p, d}-E_{F p, d-1}}{k T}\right)\right)$.

$n_{d}$ and $p_{d}$ are the unbound carrier densities while $n_{d-1}$ and $p_{d-1}$ are the bound low-dimensional well densities. $E_{F\{n, p\}\{d, d-1\}}$ are the QFLs of the corresponding populations. $N_{d-1}$ and $P_{d-1}$ are the maximum bound electron and hole densities which we determine by integrating the lowdimensional density of states obtained from the $\mathbf{k} \cdot \mathbf{p}$ calculation up to the barrier bandedge.

Capture is hence proportional to the number of unbound carriers, decreases with the filling of the bound states and increases with QFL separation. When the bound population is completely filled up, or when there is no difference in the QFLs of the populations, net capture will be zero. This is the situation demanded by a vanishing current in equilibrium. Omission of the exponential term would lead to unphysical effects as this would mean at equilibrium that either the bulk density has to vanish within the nanostructure or the bound population must be completely filled, both of which are not reflecting the physical situation.

\subsubsection{Correction of bandedges within a quantum region}

An unbound carrier within a quantum region must have at least the energy of the barrier bandedge, not of the quantum region bandedge. Hence it is necessary to substitute the well, wire or dot bandedges with the barrier bandedge in the continuity equations for the unbound carriers. This has the consequence that unbound carriers experience no more band offset when they enter the quantum region. 


\subsubsection{Fermi statistics}

For structures with degenerate doping, Fermi statistics need to be considered. For example, (5a) is in the bulk case replaced by [18]

$\mathbf{J}_{n}=\mu_{n}\left(n \nabla\left(E_{c}-e \phi\right)-\lambda^{(3)} \frac{3}{2} n k T \log m_{e}+k T \lambda^{(3)} \nabla n\right)$,

where

$\lambda^{(3)}(v)=\frac{v}{\mathcal{F}_{-1 / 2}\left(\mathcal{F}_{1 / 2}^{-1}(v)\right)}$

is a unitless function approaching 1 for $v \rightarrow 0$, evaluated at $v=\frac{n}{N_{c}}$. Note that the resulting current can still be divided in a drift and a diffusion part, with the modified Einstein relation [18] $D_{n}=\mu_{n} \frac{k T}{e} \frac{\mathcal{F}_{1 / 2}\left(\frac{E_{F}-E_{c}}{k T}\right)}{\mathcal{F}_{-1 / 2}\left(\frac{E_{F}-E_{c}}{k T}\right)}$.

\subsection{Electrostatics}

In addition to the continuity equations, the Poisson equation is solved:

$\nabla \cdot(\epsilon \nabla \phi)=e\left(p^{(t o t)}-n^{(t o t)}+N_{D}-N_{A}\right)$.

$e\left(N_{D}-N_{A}\right)$ is the net ionized dopant charge density, $\epsilon=$ $\epsilon_{s} \epsilon_{0}$ the static permittivity and $n^{(t o t)}, p^{(t o t)}$ are the total carrier densities, i.e. the sum of bulk and low-dimensional carrier densities. Incomplete dopant ionization is treated in the standard way found e.g. in [18].

The low-dimensional carrier densities are spread out in the quantized directions according to their wavefunctions:

$n_{d}\left(x_{/ /}, x_{\perp}\right)=n_{d}\left(x_{/ /}\right) \sum_{n \mathbf{k}} f_{n \mathbf{k}}\left|\psi_{n \mathbf{k}}\left(x_{\perp}\right)\right|^{2}$,

and similarly $p_{d}(d=0,1,2)$. Here the three-dimensional coordinate $x$ is split tensorially into coordinates $x_{/ /}$of the low-dimensional transport grid and $x_{\perp}$ of the lowdimensional Schrödinger grid. $f_{n \mathbf{k}}$ is an appropriate weighting of the wavefunctions $\psi_{n \mathbf{k}}$, usually the Fermi factor $\left(1+\exp \left(\frac{E_{n \mathbf{k}}-E_{F n}}{k T}\right)\right)^{-1}$ with some normalization. However a variant is also implemented where only the lowest-lying state is considered: $f_{n \mathbf{k}}=\delta_{n 0} \delta_{\mathbf{k} \mathbf{0}}$.

\subsection{Boundary conditions for the drift-diffusion equations}

We employ the standard drift-diffusion boundary conditions. For the continuity equation this means charge neutrality within a contact and Neumann conditions elsewhere. The Poisson equation has Dirichlet conditions at the contacts, where the potential is found from the densities and the fixed
Fermilevels using (3) or its Boltzmann approximation, and Neumann conditions elsewhere. Due to reasons elaborated in Sect. 5, only bulk carrier populations are contacted directly.

\subsection{A note on the quasi-Fermilevels}

In the proposed model, the drift-diffusion QFLs assuming parabolic bands do not coincide with the QFLs obtained from the $\mathbf{k} \cdot \mathbf{p}$ bandstructure using the same density. While it is consistent to use the former quantities in the transport simulation, the latter need to be employed in the luminescence calculation as well as in the factors $f_{i}$ in (11). At thermal equilibrium, the solution will only enforce the parabolicband-QFLs to be constant. A possible improvement of this model would be to fit the effective masses entering the transport equations to the $\mathbf{k} \cdot \mathbf{p}$ results, thus improving the quasiFermilevels entering the capture equations (7).

\section{Luminescence from nanostructures}

\subsection{Band structure}

Spontaneous emission spectra can be obtained from knowledge of the band structure and the carrier densities (or, equivalently, the QFLs). We employ the widely used $\mathbf{k} \cdot \mathbf{p}$ method $[15,23]$ to calculate the band structure in the vicinity of the $\Gamma$-point. Advantages over other methods are the applicability of a common formalism to all types of nanostructures, easy incorporation of strain, parameter availability for all optoelectronic materials and reasonably fast computation times such that it can be employed in a self-consistent calculation together with a transport simulation. However, standard $\mathbf{k} \cdot \mathbf{p}$ methods fail to incorporate effects from material interfaces and remote $k$-points. Details of the formulation and implementation are discussed in [24].

\subsection{Luminescence}

We employ multiband versions of the luminescence calculations using thermalized carrier densities elaborated in [25] for wells and more generally in [26]. For accurate luminescence and gain spectra, it is important to include carriercarrier interactions. We are currently working on an implementation of many-body effects on the screened HartreeFock (SHF) level which is able to describe excitonic effects as well as bandgap renormalization and phenomenological scattering. However, the results presented in Sect. 5 use freecarrier theory in which the position- and energy-resolved spontaneous emission rate is given by [25] 


$$
\begin{aligned}
& \mathcal{L}(\mathbf{x}, \hbar \omega) \\
& =\frac{e^{2} n_{r} \hbar \omega}{\pi \hbar^{2} c^{3} m_{0}^{2} \epsilon_{0}} \frac{1}{(2 \pi)^{d}} \sum_{m, n} \sum_{\mathbf{k}} M_{n m}(\mathbf{k}) \\
& \quad \times \Gamma\left(\hbar \omega, E_{n \mathbf{k}}-E_{m \mathbf{k}}\right) f\left(E_{n \mathbf{k}}, E_{F n}\right)\left(1-f\left(E_{m \mathbf{k}}, E_{F p}\right)\right) .
\end{aligned}
$$

Here $n_{r}$ is the background dielectric constant, $m_{0}$ is the bare electron mass, $m$ and $n$ denote electron and hole subbands, and $\Gamma$ is a normalized lineshape function centered around $E_{n \mathbf{k}}-E_{m \mathbf{k}}$ which we choose to be of $\cosh ^{-1}$-type. $M_{n m}(\mathbf{k})$ is the momentum matrix element between electron state $n \mathbf{k}$ and hole state $m \mathbf{k}$. Note that the spin is included in the subband indices.

The luminescence $\mathcal{L}(\mathbf{x}, \hbar \omega)$ is coupled to the continuity equation as a recombination mechanism:

$R_{\text {lumi }}(\mathbf{x})=\int_{0}^{\infty} d(\hbar \omega) \mathcal{L}(\mathbf{x}, \hbar \omega)$.

The spectrum and the total output power of the device are then found by

$$
P(\hbar \omega)=\int d \mathbf{x} \mathcal{L}(\mathbf{x}, \hbar \omega), \quad P=\int_{0}^{\infty} d(\hbar \omega) P(\hbar \omega) .
$$

\section{Numerical solution}

\subsection{Discretization of the transport problem}

The well-known Scharfetter-Gummel box-method discretization [27] is employed for the continuity equations and a finite volume discretization for the Poisson equation. The resulting equations, whose form is independent of grid dimensionality, are given in the Appendix.

\subsection{Interpolation between grids}

As the transport of bound carriers is restricted to fewer dimensions than in the bulk case, the corresponding continuity equations are also solved on different, low-dimensional grids. This creates the need to interpolate the following quantities:

- The electrostatic potential, defined on the bulk grid, must be interpolated onto the low-dimensional grids. To do so, we determine for each low-dimensional vertex its position within the bulk grid and interpolate linearly from the vertices constituting the bulk element it lies in:

$$
\phi\left(x_{d D}^{(i)}\right)=\sum_{j} \lambda_{i j} \phi\left(x_{3 D}^{(j)}\right), \quad \sum_{j} \lambda_{i j}=1 .
$$

- Low-dimensional density must be included in the Poisson equation on the bulk grid. For this it is spread out in the quantized directions with the energy-weighted sum of wavefunctions (11). The low-dimensional transport grid and the wavefunction grid compose a tensorial grid having the same dimension but not being equivalent to the bulk grid. Bulk grid vertices are written as a linear combination of tensorial vertices and receive density with the corresponding weights:

$n_{d}\left(x_{3 D}^{(i)}\right)=\sum_{j} \lambda_{i j} n_{d}\left(\left(x_{/ /}, x_{\perp}\right)^{(j)}\right), \quad \sum_{j} \lambda_{i j}=1$.

- For the capture rates (7) several low-dimensional quantities must be given on bulk vertices. For this a bulk vertex is projected orthogonally onto the low-dimensional grid and the projection is written as a linear combination of low-dimensional vertices. The value on the bulk vertex is the corresponding weighted sum of low-dimensional values.

- The captured density must be generated on the lowdimensional grid. Distribution is not straightforward, as it is important to generate the density homogeneously and at the same time ensure particle conservation:

$\sum_{i} V_{2}^{(i)} G_{2}^{(j)}(i)=V_{3}^{(j)} R_{3}(j)$,

where $i$ and $j$ are low-dimensional and high-dimensional vertices, $V_{d}^{(i, j)}$ the respective Voronoi cell sizes and $G_{2}^{(j)}(i)$ the generation at vertex $i$ arising from capture at vertex $j$. A proper treatment is cumbersome and would involve projection of high-dimensional voronoi cells onto the low-dimensional Voronoi cells and corresponding intersection calculations. Instead the following approach is used which is satisfactory for similar degrees of refinement of both grids. First the bulk vertex $j$ is projected onto the low-dimensional grid. Then all low-dimensional vertices $i^{\prime}$ which are closer to that point than to any other projected bulk vertex are determined, and the captured density is distributed between these vertices according to their Voronoi cell size:

$G_{2}^{(j)}(i)=\frac{V_{2}^{(i)}}{\sum_{i}^{\prime} V_{2}^{\left(i^{\prime}\right)}} \cdot \frac{V_{3}^{(j)}}{V_{2}^{(i)}} R_{3}(j)$

In cases where there are no low-dimensional vertices closer to the projected point than to any other projected point, the capture is given to the vertices constituting the low-dimensional element in which the projection is located. The total generation at a vertex $i$ is the sum over all bulk vertices $j$. 


\subsection{Interpolation between $\mathbf{k} \cdot \mathbf{p}$ slices}

The description of a bound carrier by its wavefunction in the quantized direction would in fact require the solution of a Schrödinger equation with varying electrostatic potential at every point of the low-dimensional transport grid. This would bring about an immense computational burden. We instead select certain points in the low-dimensional transport grid, termed $\mathbf{k} \cdot \mathbf{p}$ slices, where we perform the $\mathbf{k} \cdot \mathbf{p}$ calculation for the spreading and luminescence. The spreading shape, band structure and $\mathbf{k} \cdot \mathbf{p}$-QFLs of the remaining points are then interpolated linearly.

\subsection{Newton method for the transport equations}

The Poisson equation together with $2 q+2$ continuity equations (where $q$ is the number of quantum structures in the device) forms a system of nonlinear equations which is solved by a Newton procedure. Our implementation features an advanced dependency and timestamp concept which is completely independent of the actual solved equations. This allows fast and conceptually clear implementation of new models. The solver is able to assemble the total derivatives with respect to Newton variables entering the Jacobian using only partial derivatives and the chain rule.

The basic entity of the developed solver is an equation class which encapsulates a number of equations for the same number of solution variables of some entity. Each of these classes has a list of dependencies on other classes. The solver makes a basic distinction between explicit equations, where the solution variable can be computed directly from the variable values of the dependencies, and implicit equations where such a relation cannot be given and the equation must be solved by the Newton algorithm. Examples are the Poisson equation as an implicit equation and (5) as an explicit equation. The solution variable of the Poisson equation is the electrostatic potential, and its dependencies are the various densities as well as the electrostatic permittivity. The solution variable of (5) is the particle current and the dependencies are the band edge, mass, density, temperature and potential.

Only the partial derivatives with respect to the direct dependencies need to be implemented for each equation/variable. Implicit equation objects hence compute the Newton function and the partial derivatives with respect to all direct dependencies of the equation, explicit equations compute their own variable values and the partial derivatives with respect to all dependencies of that formula.

The mentioned automatization has virtually no computation expense as the sparsity pattern of the Jacobian matrix is determined prior to its computation. Like this only nonzero matrix entries are actually computed.

We have implemented all necessary derivatives in our equations, such that all quantities are solved simultaneously and no Gummel iteration needs to be employed. We have experienced good convergence with this method.

\subsubsection{Linear solver and employed unit system}

For the solution of the linear equation system, several solvers can be chosen from. Pardiso [28] and Umfpack [29] are freely available direct sparse system solvers. However, we observed that the Jacobian can in rare cases get very badly conditioned during the Newton iteration. In these cases it is more convenient to work with an iterative solver such as ILS [30] whose deflation of eigenvalues makes it insensitive to this problem.

A reasonable choice of physical units also improves convergence. The simulator is designed such that the unit system can be set by giving four fundamental constants for length, time, energy and charge. For quantum-sized structures, standard de Mari scaling [31] is not appropriate as the whole device is much smaller than the diffusion length. We usually choose the units $10 \mathrm{~nm}-1 \mathrm{ps}-1 \mathrm{eV}-1 \mathrm{e}$.

\subsubsection{Solution of the transport problem}

A couple of restrictions were applied to achieve convergence in the solution of the transport equations:

- An inexact line search algorithm scales down the update vector when needed such that the norm of the newly obtained right-hand side is smaller than the old one. The minimum scale is $10^{-5}$. However, especially far away from the solution this criterion is not always met.

- Densities are kept above some minimum value at all stages. While this does not change the device behaviour, it drastically improves convergence at low voltages. Typical minimum densities are $10^{-11} \mathrm{~cm}^{-3}$ for 3D densities, $10^{-6} \mathrm{~cm}^{-2}$ for $2 \mathrm{D}$ densities and $10^{-12} \mathrm{~cm}^{-1}$ for $1 \mathrm{D}$ densities.

- The electrostatic potential receives a maximum update of $\pm 0.2 \mathrm{~V}$ and the $\mathbf{k} \cdot \mathbf{p}$ QFLs a maximum update of $\pm 0.1 \mathrm{~V}$. This prevents overshoot during the first Newton iterations. - The exponential factor in (7) is limited to $e^{10}$.

The convergence criterion applied to each quantity reads

$\frac{|\Delta \mathbf{x}|}{|\mathbf{x}|+n_{x} x_{\text {ref }}}<\epsilon$.

$n_{x}$ is the number of solution variables of a given quantity, e.g. the number of vertices in case of the electrostatic potential. $\mathbf{x}$ is the vector of solution variables of the quantity and $x_{\text {ref }}$ a reference size. We typically set $x_{r e f}=0.1 \mathrm{~V}$ for potentials, $10^{20} \mathrm{~cm}^{-3}$ for a 3D density, $10^{13} \mathrm{~cm}^{-2}$ for a 2D density and $10^{7} \mathrm{~cm}^{-1}$ for a $1 \mathrm{D}$ density. $\epsilon$ is a mixture of relative and absolute error and is typically chosen as $10^{-6}$ $10^{-7}$. Please note that all of these numerical values were determined by experience. 


\subsubsection{Initial guesses}

Choosing starting values for the densities and the potential which are reasonably close to the solution is of the essence. We solve (10) in the absence of space charge to obtain an initial guess for the electrostatic potential. For the bulk and quantized densities, the majority carriers are set to the doping value whereas the minority carries are set to the intrinsic value.

\section{$4.5 \mathbf{k} \cdot \mathbf{p}$ equations}

Within this work, a stand-alone solver for $4 \times 4,6 \times 6$ and $8 \times 8$ zincblende and wurtzite problems using the finite element method has been developed. The eigenvalue problem associated with $\mathbf{k} \cdot \mathbf{p}$ envelope equations for wells, wires and dots and its numerical solution is elaborated in [24].

\subsection{Self-consistence of transport and luminescence}

The $\mathbf{k} \cdot \mathbf{p}$ calculation is an eigenvalue problem and cannot be included in the Newton solution scheme of the transport equations. It relies on the densities and the electrostatic potential and itself influences the transport equations through radiative recombination and the spreading according to (11). Therefore the transport and $\mathbf{k} \cdot \mathbf{p}$ calculations need to be iterated until self-consistence is achieved. It was shown [32] that this process may not converge unless a predictor-corrector (PC) scheme is employed in which the derivative of a quantity relying on band structure with respect to the potential is predicted, even though the band structure is kept constant in the Newton iteration. We have indeed observed that outer convergence may fail at elevated carrier densities unless the PC scheme is employed, in which case one typically needs less than 10 iterations. Although in [32] the carrier densities were expressed explicitly in terms of the potential, their findings apply to our problem as well.

\subsection{AQUA - A QUAntized structure simulator}

All of the above has been realized in a highly objectoriented $\mathrm{C}++$ code which we call AQUA. Focus was put on extendability, sustainability and clarity. Top-level program steering is done using the scripting language Python. The performance-critical sections of Jacobian and RHS assembly, linear systems solver and eigenvalue solver are parallelized. Figure 2 summarizes the simulation flow. AQUA only relies on freely available software.

\section{A quantum well-wire example}

The AlGaAs-GaAs QWR LED structure investigated experimentally in [17] provides a first validation of the applicability of our developed simulator. Choosing an AlGaAs-based

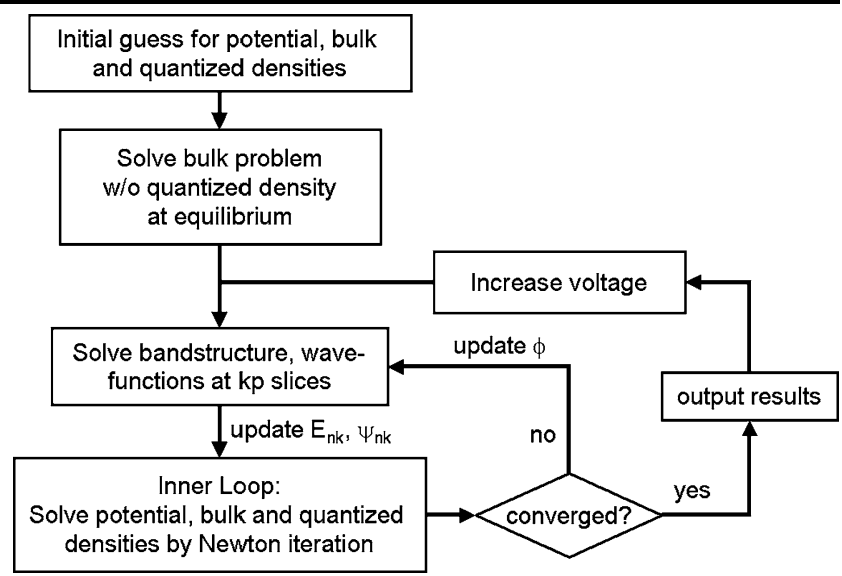

Fig. 2 Simulation flow in AQUA

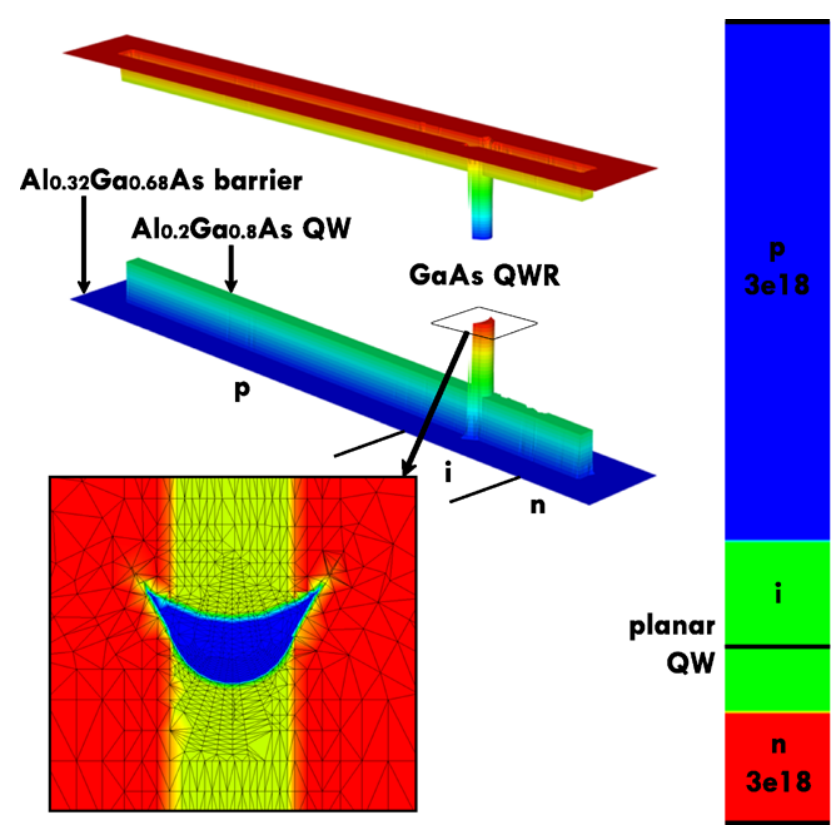

Fig. 3 Band edges (not to scale) and mesh of the QWR diode, and doping profile of the QW diode structure [17]

device facilitates the analysis because the material system has well-established parameter sets. The device consists of a V-grooved QWR embedded in a series of QWs, two of which (termed vertical QWs or VQWs) are contacted electrically. The device is expected to operate by channeling injected carriers through the VQWs and capturing them in the QWR where radiative recombination preferably takes place. Using the VQWs as channel increases the collection of electrically injected carriers by the wire and hence yields a high radiative efficiency for the wire. Our simulation results will confirm this picture.

The model of the structure is shown in Fig. 3. Compared to the experimental structure, the lateral wells are omitted as they are not expected to influence the electroluminescence experiments. We adjust the distance between the wire and 
the p-region to $90 \mathrm{~nm}$ instead of a nominal $200 \mathrm{~nm}$ due to suspected diffusion of the p-dopant zinc atoms. Like in [17], a comparison is made to a planar structure comprising a single QW, also depicted in Fig. 3. The QWR diode and the QW diode have the same doping profile.

The low-dimensional carrier populations are not contacted directly. Carriers are always first injected into bulk in our model. A combination of bulk and low-dimensional injection at the lateral ends of the QW is inherently not feasible unless one restricts the contact region to the well. This in turn would diminish the contact area and lead to an unphysically high contact resistance which would govern the behaviour of the whole device. Instead the device is preceded with highly doped bulk regions, and injection into the ends of quantum structures will happen by itself via capture.

The lack of contacts in the low-dimensional continuity equations brings with it the inability of low-dimensional carriers to escape the structure other than through recombination or reemission into bulk. This is unphysical since QW carriers there should experience no additional energy barrier when leaving the structure. We circumvent this obstacle by introducing an artificial recombination of the form $\frac{n-n_{i}}{\tau}$ for minority carriers with very short recombination times. This forces a carrier which has traveled along the whole quantum well to recombine at the end. The associated recombination current is added to the contact current of the corresponding side. This provides a sufficient description of the experimental situation, also considering that not much is known about the precise mechanisms of injection and removal at the end of quantum regions.

\subsection{Simulation setup}

The dielectric permittivity is modeled as regionwise constant, and the Arora model [33] is employed for the dopingand temperature-dependent mobility with binary parameters and alloy interpolation as in [34]. Carrier or lattice heating should be negligible, and electric fields are not so high as to significantly change the mobility. Dopants are assumed to be completely ionized. The transport simulation uses Boltzmann statistics. Equations (7) are employed for bulk-well, bulk-wire and well-wire capture. Renormalization of bandgaps due to many-body effects is not included. For the temperature dependence of the alloy bandgap, we take the Varshni bandgaps of the constituting binary materials and interpolate with a temperature-independent bowing parameter, thus not relying on interpolations of the Varshni parameters (the same methodology is used in [16]). The entire bowing takes place in the conduction band. Simulation parameters not listed in Table 2 are taken from [35].

For the $\mathbf{k} \cdot \mathbf{p}$ calculations, the six-band model was used with $12 \mathrm{k}$-points between $k=0$ and $k=1.5 \mathrm{~nm}^{-1}$. The well band structure was solved in radial approximation. 17 points
Table 2 Simulation parameters unless listed in [35]

\begin{tabular}{ll}
\hline Parameter & Value (Al molefraction $x<0.4)$ \\
\hline$m_{h} / m_{0}$ & $0.4809+0.29 x$ \\
$\mu_{n, p}$ & {$[34]$} \\
$\epsilon_{s}$ & $12.9-2.84 x-0.625 x(1-x)[34,36]$ \\
$E_{P}[\mathrm{eV}]$ & $18.0+3.1 x[23,35]$ \\
$\tau_{n, p}^{\max }$ & $10^{-9} \mathrm{~s} ;$ wire: $10^{-10} \mathrm{~s}$ \\
$N_{r e f}^{S R H}$ & $10^{16} \mathrm{~cm}^{-3}$ \\
$c_{n, p}^{\text {Aug }}$ & $10^{-30} \mathrm{~cm}^{6} \mathrm{~s}^{-1}$ \\
$\tau_{\text {bulk } \rightarrow \text { well }}$ & see text \\
$\tau_{\text {bulk } \rightarrow \text { wire }}$ & $10^{-6} \mathrm{~s}$ \\
$\tau_{\text {well } \rightarrow \text { wire }}$ & see text \\
\hline
\end{tabular}

of the drift-diffusion grid of the 2D well carriers were selected along the transport direction where a $\mathbf{k} \cdot \mathbf{p}$ problem was solved. For the wire, one $\mathbf{k} \cdot \mathbf{p}$ slice sufficed due to the translational invariance of the structure in the wire transport direction. A maximum of 28 bound valence subbands and 8 bound conduction subbands was encountered in the well problems. The wire displayed 12 bound electron subbands and over 30 bound valence subbands.

\subsection{Solution procedure}

To have a coherent methodology for the determination of unknown system parameters, the planar QW diode $I-V$ curves were investigated first to check the used literature parameters and determine the temperature-dependent well capture times. ${ }^{1}$ Then the QWR diode was simulated and the wire capture times fitted to experimental results. We included an additional series resistance of $10 \Omega$ (compared to a diode turn-on resistance of 500-800 $\Omega$ ). Using this procedure, we obtain bulk-well capture times of $\left\{3 \cdot 10^{-12}, 8 \cdot 10^{-12}\right.$, $\left.2 \cdot 10^{-11}, 8 \cdot 10^{-11}\right\} \mathrm{s}$ from the QW diode simulation and well-wire capture times of $\left\{3 \cdot 10^{-13}, 2 \cdot 10^{-12}, 8 \cdot 10^{-12}\right.$, $\left.4 \cdot 10^{-11}\right\}$ s from the QWR diode simulation, both for temperatures of $\{300,240,175,90\} \mathrm{K}$. There is little discussion of such a temperature dependence in literature. However, an increase of the capture time seems plausible as both electron-electron and electron-phonon scattering mechanisms freeze out at low temperatures.

We found that using Fermi statistics or incomplete dopant ionization as well as different $\mathbf{k} \cdot \mathbf{p}$ models and mobilities has almost no effect on the QW $I-V$ curves. The parameters governing the $I-V$ characteristics below turn-on are the

\footnotetext{
${ }^{1}$ The contact area of the simulated QWR diode is $10 \times 100 \mathrm{~nm}^{2}$, $10 \mathrm{~nm}$ being the extension in the wire transport direction. To compare our results with [17], extensive quantities were scaled by a factor of $(250 \mu \mathrm{m} / 10 \mathrm{~nm}) \cdot 500=1.25 \cdot 10^{6}$ for the QWR diode and $(250 \mu \mathrm{m})^{2} /(10 \mathrm{~nm} \cdot 100 \mathrm{~nm})=6.25 \cdot 10^{7}$ for the QW diode.
} 


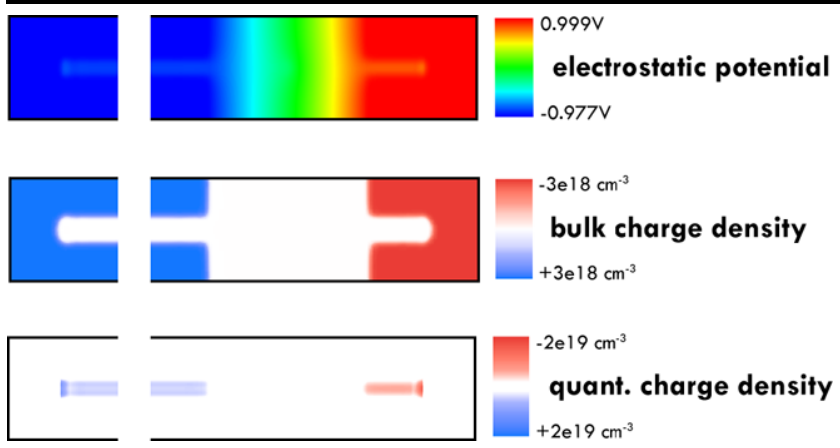

Fig. 4 QWR diode equilibrium electrostatic potential and carrier densities at $T=175 \mathrm{~K}$

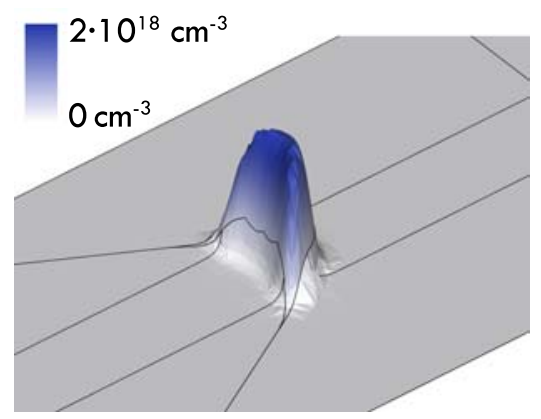

Fig. 5 Room temperature hole density of the bound wire population at $V_{t o}$

capture time, which determines how efficiently carriers are transferred into the bound population, and the SRH lifetime. Recombination current dominates over drift and diffusion.

The system was first solved without low-dimensional densities for convergence reasons. For low-temperature simulations, the temperature was ramped from $300 \mathrm{~K}$ down to the desired value in steps of $50 \mathrm{~K}$. Then the voltage was ramped starting from equilibrium up to $1.6-2.0 \mathrm{~V}$ depending on the temperature.

Attempts to include the well density in the electrostatics according to (11) in the QWR diode were unsuccessful because of the high occupation in the doped zones even at equilibrium. Instead we employed the lowest flat-band state for the transversal distribution of the well carriers. For the luminescence and all wire-related matters the full $\mathbf{k} \cdot \mathbf{p}$ results were used.

\subsection{Transport}

The electrostatic potential, bulk and bound densities at thermal equilibrium are shown in Fig. 4. Within the VQWs the unbound population is almost depleted whereas the bound population is present up to the very end of the well. The sum of the populations does not exactly compensate the doping, such that some space charge remains which is visible in the potential. The wire population located in the centre of the

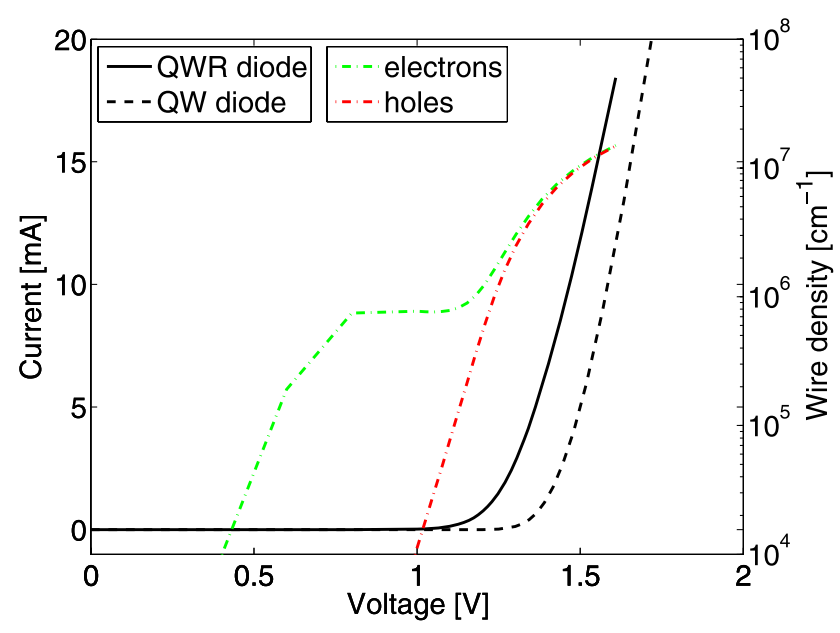

Fig. 6 Room temperature current-voltage characteristics and behaviour of the bound wire populations

i-region is too low to be visible in Fig. 4. We instead plot a typical hole density at room temperature and turn-on voltage in Fig. 5. The shape of the density is determined according to (11) where the quasi-Fermilevel is determined from the $\mathbf{k} \cdot \mathbf{p}$ results and the drift-diffusion density. As the shape is a mixture of populated subbands, the distribution does not resemble any particular $\mathbf{k} \cdot \mathbf{p}$ state. The bound electron density closely resembles the hole density except for a potentialinduced shift to the $\mathrm{n}$-side.

Figure 6 shows obtained current-voltage characteristics at room temperature for the QWR and the QW diode together with the one-dimensional bound electron and hole densities of the wire. The curves are dominated by the exponential behaviour arising from the pn-junction potential barrier. They can be approximated by

$I=I_{0} \exp \left(\frac{e V}{n_{f} k T}\right)$

$I_{0}$ being the dark current and $n_{f}$ the ideality factor of the device. An ideal pn-diode has an ideality factor of 1 whereas recombination currents from SRH, Radiative and Auger recombination typically have ideality factors of 2,1 and $2 / 3$, respectively. In [17] it was only noted that $n_{f}$ is similar for both the QW and the QWR diodes whilst not giving an explicit value. In our simulations we encounter $n_{f} \sim 2$ for voltages below the turn-on voltage for both the QW and QWR diode, even at low temperatures where radiative recombination dominates.

The asymmetric behaviour of the two bound populations at low voltages seems to arise from the different potential barriers between the wire and the $\mathrm{n}$ - and $\mathrm{p}$-doped regions. After a quick increase, the electron density reaches a plateau at around $1 \mathrm{~V}$. During this phase, a voltage increase effectively only lowers the potential barrier to the $\mathrm{p}$-side until the 


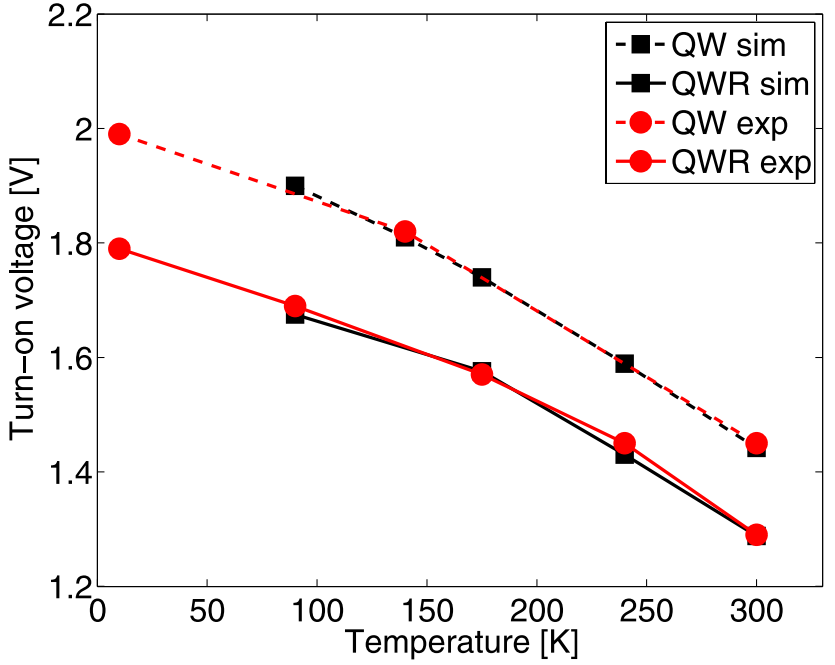

Fig. 7 Experimental and simulated turn-on voltages

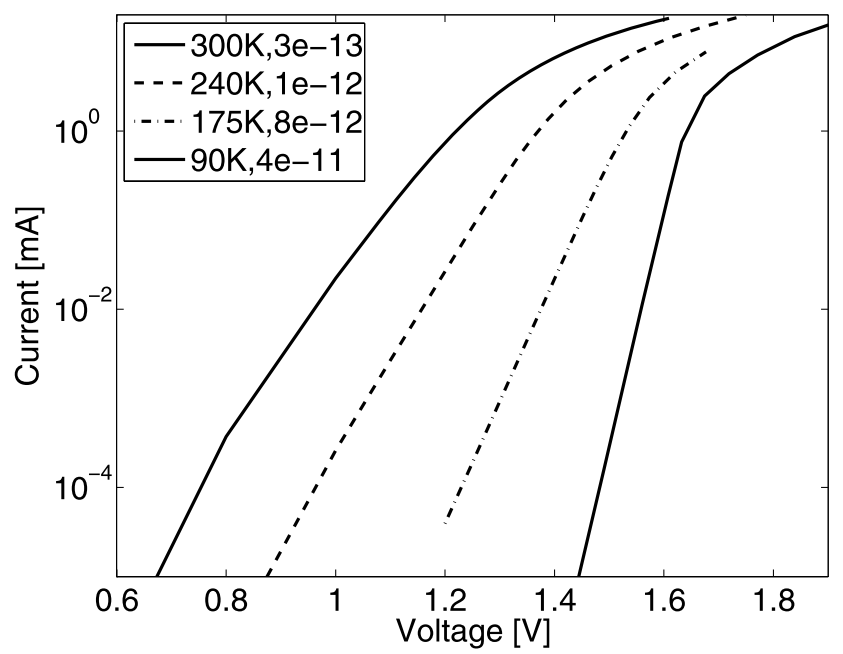

Fig. 8 QWR diode $I-V$ characteristics at different temperatures (logarithmic $I$-scale)

electron and hole densities reach the same order of magnitude. A further voltage increase then lowers both energetic barriers by equal amounts.

The turn-on voltage $V_{t o}$ is defined at $I_{t o}=2.5 \mathrm{~mA}$. Above this point the wire density starts to saturate, as seen in Fig. 6, and carrier capture gets inefficient. The bound wire density at turn-on is in the range of a few $10^{6} \mathrm{~cm}^{-1}$, in agreement with the findings of [17]. A comparison of experimental and simulated temperature dependencies of $V_{t o}$ is shown in Fig. 7. The potential barrier in the QWR diode is lowered by $100-200 \mathrm{mV}$ compared to the QW diode, thus indicating that the carriers are indeed channeled through the lower-bandgap well. This finding is supported by an analysis of the current densities (not shown) confirming that current is transferred from the bulk to the quantized well population.

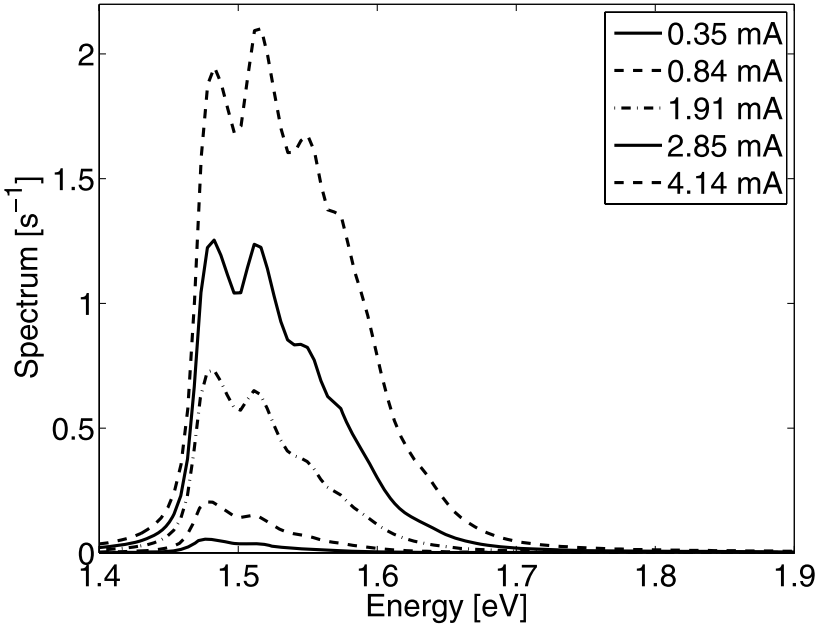

Fig. 9 Combined wire-well luminescence at room temperature and different currents

Current-voltage characteristics for different temperatures are shown in Fig. 8. From a fit to (20) up to the turn-on voltage with $n_{f}=2$ we find dark currents of $\left\{3 \cdot 10^{-49}\right.$, $\left.2 \cdot 10^{-25}, 8 \cdot 10^{-18}, 8 \cdot 10^{-14}\right\}$ A for the QWR diode and $\left\{10^{-55}, 3 \cdot 10^{-28}, 5 \cdot 10^{-20}, 10^{-15}\right\}$ A for the QW diode at $\{90,175,240,300\} \mathrm{K}$, although the curves do not follow closely an exponential behaviour in the QW case. This confirms the statement in [17] that dark currents are some 2-3 orders of magnitude higher in the QWR diode.

As the total device length of only $0.7 \mu \mathrm{m}$ between the contacts is much smaller than the diffusion length, minority carriers do not experience enough recombination to return to their equilibrium value before they reach the opposite contact. Below $V_{t o}$ most of the injected carriers are captured into low-dimensional densities and current is dominated by the recombination current of the highest quantized population, which is in this case the bound wire population. We found that in order to achieve the measured values of $V_{t o}$ at room temperature, SRH recombination rates of $10^{10} \mathrm{~s}^{-1}$ in the wire and $10^{9} \mathrm{~s}^{-1}$ elsewhere had to be assumed. (Note that this value will get modified with the local doping according to (6) for the actual SRH rate.) Nonradiative recombination dominates over radiative recombination at room temperature but not at lower temperatures. High above saturation, the current is dominated by the artificial recombination current of well carriers traveling across the wire without being captured, as explained earlier in this section.

Finally we point out that the magnitude of bound wire density is dominated by capture and recombination times. The bound well density is mainly governed by electrostatics because the well is situated along a p-i-n junction.

\subsection{Luminescence}

Figure 9 shows the room-temperature luminescence spectrum arising from the combined wire-well system at differ- 

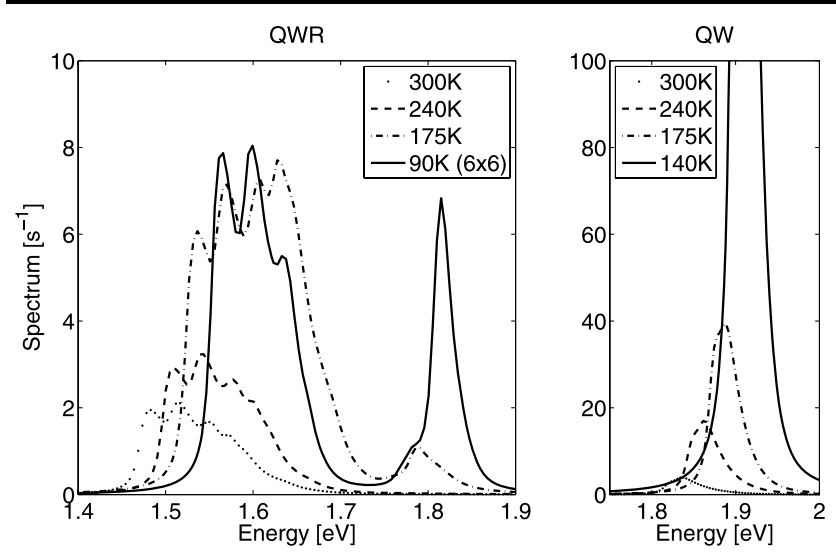

Fig. 10 Left: Combined wire-well luminescence in the QWR diode at $V_{t o}$ for different temperatures. Right: QW diode

ent currents, although the well emission is too weak to be seen on a linear scale plot. Total emission from the wire saturates around the turn-on voltage. Different peaks according to transitions of different subbands are observed at this point. The spacing of around $30 \mathrm{meV}$ between the peaks qualitatively agrees with the experimental observations. It is determined by the conduction subbands which are much wider spaced than the valence subbands. Contrary to the statement in [17], we find that transitions between subbands with different index numbers do influence the emission spectrum. The FWHM is not discussed here since it would be significantly modified by inclusion of many-body effects in the luminescence calculations. Given the uncertainty about the structure geometry and material composition, these results reproduce the findings in [17] to a satisfying extent.

In Fig. 10 the temperature dependence of the luminescence is shown. An analysis shows that the relative magnitude of the nonradiative recombination mechanisms in the wire compared to the radiative recombination decreases from a factor of 25 at room temperature to about 1 at $90 \mathrm{~K}$. Thus nonradiative recombination plays a significant role at these temperatures. However, at even lower temperatures radiative emission would dominate, indicating a high internal quantum efficiency of the device especially at cryogenic temperatures.

\section{Conclusion and outlook}

We have introduced the reader to a new simulation tool capable of investigating transport and luminescence in combined well-wire-dot nanostructures. The underlying model is based on the partition of space into different quantization degrees and on the distinction between bound and unbound populations. Bound populations are described in a purely quantum-mechanical way in the directions of confinement and in a purely classical way in the lateral directions. Movement of bound carriers is governed by drift and diffusion and only takes place in the lateral directions. Populations are coupled by capture and the electrostatic potential.

The applicability of the developed approach was tested with a QWR LED structure consisting of a wire connected to wells. Experimental results could be explained to a reasonable degree, suggesting that all the essential physics of both transport and light emission in this structure was captured. Simulations indicate a large dependence of the capture times on the temperature.

Our tool offers promising applications in future academic research on a wide class of nanostructures, such as nitride light emitters, nanowire solar cells, quantum dot, wire and well LEDs. The modularity of the code allows the concise implementation of different physical models with high degrees of complexity. When coupled with an optical simulation, the tool also has the potential to investigate laser devices such as VCSELs [37]. Furthermore, the present model could serve as a fundament for full quantum simulations on a small but pivotal central part of the structure, thus facilitating the investigation of large nonplanar optoelectronic devices on a sound theoretical footing.

Acknowledgements The authors wish to thank Simon Brugger, Martin Frey and Hektor Meier for fruitful discussions. This work was supported by the Swiss National Science Foundation through GRANT Nr. 200021-107932.

\section{Appendix: Discretized device equations}

For each grid vertex $i$, the box-discretized bulk driftdiffusion equations read [27]

$\sum_{E(i j)} \sum_{j(i)} \epsilon_{E} \frac{s_{i j E}}{L_{i j}}\left(\phi_{i}-\phi_{j}\right)-V_{i} e\left(p_{i}-n_{i}+N_{D i}-N_{A i}\right)=0$,

$$
\begin{aligned}
\sum_{E(i j)} \sum_{j(i)} \frac{s_{i j E} \mu_{n}^{E} \frac{k T}{e}}{L_{i j}} & \left(n_{i} B\left(\frac{e\left(\phi_{i}-\phi_{j}\right)}{k T}\right)\right. \\
& \left.-n_{j} B\left(\frac{e\left(\phi_{j}-\phi_{i}\right)}{k T}\right)\right)+V_{i} R_{i}=0,
\end{aligned}
$$

$$
\begin{aligned}
\sum_{E(i j)} \sum_{j(i)} \frac{s_{i j E} \mu_{p}^{E} \frac{k T}{e}}{L_{i j}} & \left(-p_{i} B\left(\frac{e\left(\phi_{j}-\phi_{i}\right)}{k T}\right)\right. \\
& \left.+p_{j} B\left(\frac{e\left(\phi_{i}-\phi_{j}\right)}{k T}\right)\right)+V_{i} R_{i}=0 .
\end{aligned}
$$


$j(i)$ herein denotes all vertices $j$ connected to vertex $i$ and $E(i j)$ all elements around edge $i j . s_{i j E}$ is the part of the Voronoi surface at edge $i j$ which lies in element $E(i j), L_{i j}$ is the length of edge $i j, \mu_{n, p}^{E}$ are the electron and hole mobilities in element $E$ and $B(x)=\frac{x}{e^{x}-1}$ is the Bernoulli function. Written in this form, the equations are applicable to problems of any dimensionality.

For each quantized region an additional pair of equations of the form of (22) is solved on a low-dimensional grid describing the movement of the bound carriers.

\section{References}

1. Ferry, D.K.: Quo vadis nanoelectronics? Phys. Stat. Sol. (C) 5, 17-22 (2008)

2. Tian, B., Zheng, X., Kempa, T.J., Fang, Y., Yu, N., Yu, G., Huang, J., Lieber, C.M.: Coaxial silicon nanowires as solar cells and nanoelectronic power sources. Nature 449, 885-889 (2007)

3. Choi, H.J., Johnson, J.C., He, R., Lee, S.K., Kim, F., Pauzauskie, P., Goldberger, J., Saykally, R.J., Yang, P.: Self-organized gan quantum wire uv lasers. J. Phys. Chem. B 107, 8721-8725 (2003)

4. Rafailov, E.U., Cataluna, M.A., Sibbett, W.: Mode-locked quantum-dot lasers. Nature Phot. 1, 395-401 (2007)

5. Noda, S.: Seeking the ultimate nanolaser. Science 314, 260-261 (2006)

6. Robledo, L., Elzerman, J., Jundt, G., Atatüre, M., Högele, A., Fält, S., Imamoglu, A.: Conditional dynamics of interacting quantum dots. Science 320, 772-775 (2008)

7. Liu, Y., Neophytou, N., Klimeck, G., Lundstrom, M.S.: Bandstructure effects on the performance of iii-v ultrathin-body mosfets. IEEE Trans. Electron. Dev. 55, 1116-1122 (2008)

8. Aeberhard, U., Morf, R.H.: Microscopic non-equilibrium theory of quantum well solar cells. Phys. Rev. B 77, 125,343 (2008)

9. Bufler, F.M., Gautschi, R., Erlebach, A.: Monte carlo stress engineering of scaled (110) and (100) bulk p-mosfets. IEEE Electr. Device L. 29, 369-371 (2008)

10. Girndt, A., Jahnke, F., Knorr, A., Koch, S.W., Chow, W.W.: Multiband bloch equations and gain spectra of highly excited ii-vi semiconductor quantum wells. Phys. Stat. Sol. (B) 202, 725-739 (1997)

11. Kira, M., Jahnke, F., Hoyer, W., Koch, S.W.: Quantum theory of spontaneous emission and coherent effects in semiconductor microstructures. Prog. Quant. Electron. 23, 189-279 (1999)

12. Loeser, M., Witzigmann, B.: Multi-dimensional electro-optothermal modeling of broadband optical devices. IEEE J. Quant. Electron. 44, 505-514 (2008)

13. Datta, S.: Electronic Transport in Mesoscopic Systems. Cambridge University Press, Cambridge (1995)

14. Weetman, P., Wartak, M.S.: Wigner function modeling of quantum well semiconductor lasers using classical electromagnetic field coupling. J. Appl. Phys. 93, 9562-9575 (2003)

15. Kane, E.O.: Energy band theory. In: Paul, W. (ed.) Handbook on Semiconductors, vol. 1, pp. 194-217. North Holland, Amsterdam (1982)

16. Trellakis, A., Zibold, T., Andlauer, T., Birner, S., Smith, R.K., Morschl, R., Vogl, P.: The 3d nanometer device project nextnano: Concepts, methods, results. J. Comput. Electron. 5, 285-289 (2006)
17. Weman, H., Martinet, E., Rudra, A., Kapon, E.: Selective carrier injection into v-groove quantum wires. Appl. Phys. Lett. 73, 2959 (1998)

18. Smith, R.A.: Semiconductors. Cambridge University Press, Cambridge (1978)

19. Piprek, J.: Semiconductor Optoelectronic Devices: Introduction to Physics and Simulation. Academic Press, San Diego (2003)

20. Schroeder, D.: Modelling of Interface Carrier Transport for Device Simulation. Springer, Berlin (1994)

21. Wu, C.M., Yang, E.S.: Carrier transport across heterojunction interface. Solid-State Electron. 22, 241-248 (1979)

22. Grupen, M., Hess, K.: Simulation of carrier transport and nonlinearities in quantum-well laser diodes. IEEE J. Quant. Electron. 34(1), 120-140 (1998)

23. Veprek, R.G., Steiger, S., Witzigmann, B.: Ellipticity and the spurious solution problem of k p envelope equations. Phys. Rev. B 76, 165,320 (2007)

24. Veprek, R.G., Steiger, S., Witzigmann, B.: Reliable kp band structure calculation for nanostructures using finite elements. J. Comput. Electron. (2008, this issue)

25. Chuang, S.L.: Physics of Optoelectronic Devices. Wiley, New York (1995)

26. Haug, H., Koch, S.W.: Quantum Theory of the Optical and Electronic Properties of Semiconductors. World Scientific, Singapore (2004)

27. Kerkhoven, T.: On the scharfetter-gummel box-method. In: S. Selberherr, H. Stippel, E. Strasser (eds.) Simulation of Semiconductor Devices and Processes, vol. 5, p. 237 (1993)

28. Schenk, O., Gaertner, K.: Solving unsymmetric sparse systems of linear equations with pardiso. J. Future Gen. Comp. Syst. 20, 475487 (2004)

29. Davis, T.A.: Algorithm 832: Umfpack, an unsymmetric-pattern multifrontal method. ACM Trans. Math. Softw. 30, 196-199 (2004)

30. Roellin, S.: Parallel iterative solvers in computational electronics. PhD Thesis ETH No. 15859. Hartung-Gorre, Konstanz (2005)

31. de Mari, A.: An accurate numerical steady-state one-dimensional solution of the p-n junction. Solid-State Electron. 11, 33-58 (1968)

32. Trellakis, A., Galick, A.T., Pacelli, A., Ravaioli, U.: Iteration scheme for the solution of the two-dimensional schroedingerpoisson equations in quantum structures. J. Appl. Phys. 81, 7880 7884 (1997)

33. Arora, N.D., Hauser, J.R., Roulston, D.J.: Electron and hole mobilities in silicon as a function of concentration and temperature. IEEE Trans. Electron Devices 29, 292-295 (1982)

34. Sotoodeh, M., Khalid, A.H., Rezazadeh, A.: Empirical low-field mobility model for iii-v compounds applicable in device simulation codes. J. Appl. Phys. 87, 2890-2900 (2000)

35. Vurgaftman, I., Meyer, J.R., Ram-Mohan, L.R.: Band parameters for iii-v compound semiconductors and their alloys. J. Appl. Phys. 89, 5815-5875 (2001)

36. Adachi, S.: Properties of Group-IV, II-V and II-VI Semiconductors. Wiley, New York (2005)

37. Witzigmann, B., Bäcker, A., Odermatt, S.: Physics and simulation of vertical-cavity surface-emitting lasers. J. Comput. Theoret. Nanosci. 5, 1-14 (2007) 